\title{
ON BOREL MAPPINGS AND BAIRE FUNCTIONS
}

\author{
BY
}

R. W. HANSELL

\begin{abstract}
This paper studies conditions under which classes of Borel mappings (i.e., mappings such that the inverse image of open sets are Borel sets) coincide with certain classes of Baire functions (i.e., functions which belong to the smallest family containing the continuous functions and closed with respect to pointwise limits). Generalizations of the classical Lebesgue-Hausdorff and Banach theorems are obtained for the class of mappings which we call " $\sigma$-discrete". These results are then applied to the problem of extending Borel mappings over Borel sets, and generalizations of the theorems of Lavrentiev and Kuratowski are obtained.
\end{abstract}

1. Introduction. Let $X$ be a topological space and $Y$ a metric space. By the family of Baire functions from $X$ to $Y$ [7, p. 294] (also called the analytically representable functions $[9$, p. 392]) we mean the smallest family of mappings $(1)$ from $X$ to $Y$ containing the continuous mappings, and closed with respect to pointwise limits of sequences of mappings belonging to it. We resolve this family into Baire classes $\Phi_{\alpha}=\Phi_{\alpha}(X, Y)$, for $0 \leq \alpha<\omega_{1}\left(\omega_{1}=\right.$ first uncountable ordinal), by defining

$\Phi_{0}=$ family of all continuous mappings, and

$\Phi_{\alpha}=$ family of all limits of pointwise convergent sequences of mappings from $\cup_{\beta<\alpha} \Phi_{\beta}(\alpha>0)$.

By a Borel measurable mapping of class $\alpha\left(0 \leq \alpha<\omega_{1}\right)$ we mean a mapping $f: X \rightarrow Y$ such that $f^{-1}(G)$ is a Borel set of additive class $\alpha$ in $X$ whenever $G$ is an open set in $Y$ (see $\S 2$ below).

Now, it can be shown (see (5) of $\S 2$ below) that the Baire functions of class $\Phi_{\alpha}$ are Borel (measurable) mappings of class $\alpha$, respectively $\alpha+1$, according as $\alpha$ is a finite or infinite ordinal. The object of the present paper is to determine under what general conditions the converse is true. Results of this type would be potentially useful in establishing properties of Borel mappings by transfinite induction over the class of the mapping, beginning with the usually amenable case of continuous mappings.

However, as it turns out, to require that the Borel mappings of class 1 be representable as limits of continuous mappings is exceptionally strong, and

Received by the editors September 25, 1972 and, in revised form, May 14, 1973.

AMS (MOS) subject classifications (1970). Primary 54H05, 26A21; Secondary 28 A05.

Key words and phrases. Borel measurable mappings, Baire functions, Borel classifications, Baire classifications, $\boldsymbol{\sigma}$-discrete mappings.

(1) We use the terms "mapping" and "function" synonymously, although it seems traditional to speak of Baire functions. 
imposes heavy restrictions on the range space $Y .(2)$ The classical result in this regard is the following:

Lebesgue-Hausdorff theorem (see [9, p. 393]). If $Y$ is the closed unit interval I (or, more generally, if $Y=I^{m}$ where $m$ is any cardinal $\leq \aleph_{0}$ ), then the class $\Phi_{\alpha}$ coincides with the family of Borel mappings of class $\alpha$, respectively $\alpha+1$, according as $\alpha$ is finite or infinite.

Here, the domain space $X$ is assumed to be metrisable; but this can be weakened to the assumption that $X$ be perfectly normal [9, p. 133], since the key ingredient for the exceptional case when $\alpha=1$ is that $Y$ have the "extension property" [8, p. 34] with respect to $X$ (i.e., every continuous mapping defined on a closed subset of $X$ into $Y$ can be extended continuously over all of $X$ ). And, for any normal space $X$, the space $I^{m}$ has the extension property as a consequence of the theorem of Tietze.

In $\$ 5$ below (Theorem 8) we prove a generalization of the Lebesgue-Hausdorff theorem, showing that the theorem continues to hold when $Y$ is any metric space having the extension property for $X$, provided we assume the mappings are " $\sigma$ discrete" (see $\$ 3$ for the definition of this). The $\sigma$-discrete mappings were introduced in [5] for the class of metrisable spaces, where we showed they play a fundamental role in the theory of Borel measurable mappings for nonseparable metric spaces. In $\$ 3$ below we extend the definition to include general topological spaces; and we generalize some of the basic properties of $\sigma$-discrete mappings proven in [5]. This makes possible the above generalization (and another to be discussed).

The exceptional case when $\alpha=1$ (requiring that $Y$ have the extension property for $X$ ) can be circumvented, as was done by Banach in [2], merely by starting with the Borel maps of class 1 . That is, we redefine the analytically representable functions by the classes $\Phi_{\alpha}^{*}=\Phi_{\alpha}^{*}(X, Y)$, now with $1 \leq \alpha<\omega_{1}$, where

$\Phi_{1}^{*}=$ family of all Borel maps of class 1 , and

$\Phi_{\alpha}^{*}=$ family of all limits of pointwise convergent sequences of mappings from $\cup_{\beta<\alpha} \Phi_{\beta}^{*}(\alpha>1)$.

Again, it can be shown that the mappings belonging to the family $\Phi_{\alpha}^{*}$ are Borel mappings of class $\alpha$, respectively $\alpha+1$, depending on whether $\alpha$ is finite or infinite; and we seek conditions under which the converse is true. The classical result in this case is the following:

Banach Theorem ([2]; see also [9, p. 394]). Let $X$ and $Y$ be metric spaces, and assume that $Y$ is also separable. Then the class $\Phi_{\alpha}^{*}$ coincides with the family of Borel mappings of class $\alpha$, respectively $\alpha+1$, according as $\alpha$ is finite or infinite.

(2) For example, if $Y$ is the two point discrete space $\{0,1\}$, then the characteristic function of a single point of the space of real numbers is a Borel mapping of class 1 , but it is not the limit of any convergent sequence of continuous mappings (with values in $Y$ ); cf. [9, Remark on p. 391]. 
Banach's proof of this theorem (see [2]) is given in the format of a sequence of eight lemmas (Hilfssätzen), almost all of which employ a separate use of the separability of $Y$. Using the basic properties of $\sigma$-discrete mappings and some auxiliary lemmas of our own, we show that Banach's proof can be generalized (for the class of $\sigma$-discrete mappings) to the case when $Y$ is an arbitrary metric space and $X$ is a topological space such that each open set is an $F_{\sigma}$ set (i.e., a union of countably many closed sets). (See Theorem 7 of $\$ 5$ below.)

The paper concludes with some applications of the above results to the problem of extending Borel mappings over Borel sets, and, in particular, we obtain a generalization of the theorem of Lavrentiev (Theorem 10 of $\$ 6$ ).

2. Borel sets and Borel mappings. If $X$ is a topological space, we recall that the Borel sets of $X$ comprise the smallest family of subsets of $X$ containing the open (or closed) sets, and closed under the operations of complementation and countable unions. The Borel sets of $X$ are classified as $G_{\alpha}$ sets or $F_{\alpha}$ sets $\left(\alpha<\omega_{1}\right)$ as follows:

$G_{0}=$ family of all open sets;

$G_{\alpha+1}=$ family of all unions (respectively intersections) of countable collections of sets from $G_{\alpha}$ when $\alpha$ is odd (respectively even);

$G_{\lambda}=$ family of all unions of countable collections of sets from $\cup_{\alpha<\lambda} G_{\alpha}, \lambda$ a limit ordinal; and

$F_{\alpha}=$ family of all complements (with respect to $X$ ) of sets belonging to $G_{\alpha}$.

The following fact is well-known [4, p. 133].

(1) If $X$ has the property that $G_{0} \subset F_{1}$ (i.e., the open sets are $F_{\sigma}$ sets; or equivalently, the closed sets are $G_{\delta}$ sets), then $F_{\alpha} \subset G_{\alpha+1} \subset F_{\alpha+2}$, for every $\alpha<\omega_{1}$, and $\cup\left\{F_{\alpha}\right\}=\bigcup\left\{G_{\alpha}\right\}$ is exactly the family of Borel sets of $X$.

Throughout this paper, all topological spaces are assumed to satisfy the property $G_{0} \subset F_{1}$; for example, this holds when the space is metrisable or, more generally, perfectly normal.

We will need the following lemma.

Lemma 1. Let $\left\{U_{d} \mid d \in D\right\}$ be a family of disjoint open subsets of a space $X$. If $B_{d} \subset U_{d}$ is of class $G_{\alpha}$ in $X$ for each $d \in D$, then $\cup B_{d}(d \in D)$ is also of class $G_{\alpha}$ in $X .(C f .[9, p .358$, Theorem 1].)

Proof. For the class $G_{0}$ the lemma states a familiar property of open sets. Thus assume the lemma holds for all ordinals $<\alpha$, for some countable ordinal $\alpha>0$. We first suppose that $\alpha=\beta+1$, for some ordinal $\beta$. Then, assuming the hypothesis as stated, we have either (i) $B_{d}=\cup_{n=1}^{\infty} B_{d n}$ or (ii) $B_{d}=\bigcap_{n=1}^{\infty} B_{d n}$, where each $B_{d n}$ is of class $G_{\beta}$; and in the latter case we may assume also that $B_{d n} \subset U_{d}$ for each $d \in D$. By the inductive hypothesis, each of the sets $B_{n} \equiv \cup_{d \in D} B_{d n}$ is of class $G_{\beta}$. But clearly $\cup_{d \in D} B_{d}=\cup_{n=1}^{\infty} B_{n}$ in the case of (i) above, and $\cup_{d \in D} B_{d}=\bigcap_{n=1}^{\infty} B_{n}$ similarly in the case of (ii), due to the fact that the family $\left\{U_{d}\right\}$ is disjoint. Hence, the stated conclusion follows in the case when $\alpha$ is a nonlimit ordinal. 
On the other hand, if $\alpha$ is a limit ordinal, we can choose ordinals $\alpha_{1}<\alpha_{2}$ $<\cdots$ so that $\alpha=\sup \left\{\alpha_{n}\right\}$. Then, each $B_{d}$ can be written as a union of a countable family $\left\{B_{d n}\right\}$ where each $B_{d n}$ is class $G_{\alpha_{n}}$. Consequently, $\cup_{d \in D} B_{d n}$ is of class $G_{\alpha_{n}}$, by the inductive hypothesis, and

$$
\bigcup_{d \in D} B_{d}=\bigcup_{n=1}^{\infty} \bigcup_{d \in D} B_{d n} \in G_{\alpha}
$$

as required.

The lemma now follows by transfinite induction. (See Lemma 2 of $\$ 3$ below for the corresponding property for sets of class $F_{\alpha}$.)

If $B \subset X$ is of class $G_{\alpha}$ when $\alpha$ is even, or of class $F_{\alpha}$ when $\alpha$ is odd, then $B$ is said to be of additive class $\alpha$ in $X$. Similarly, if $B \in F_{\alpha}$ when $\alpha$ is even, or $B \in G_{\alpha}$ when $\alpha$ is odd, then $B$ is of multiplicative class $\alpha$. The sets of additive (respectively multiplicative) class $\alpha$ are closed with respect to unions (respectively intersections) of countable families. A subset which is both of additive and multiplicative class $\alpha$ (in $X$ ) is said to be of ambiguous class $\alpha$. Note that the members of $G_{\alpha}$ or $F_{\alpha}$ are of ambiguous class $\beta$ for all $\beta>\alpha$ by (1) above.

Let $X$ and $Y$ be topological spaces (satisfying $G_{0} \subset F_{1}$ ) and $f: X \rightarrow Y$ a mapping. We call $f$ a Borel mapping of class $\alpha$ (or simply a mapping of class $\alpha$ ) if for every open set $G \subset Y, f^{-1}(G)$ is of additive class $\alpha$ in $X$ (equivalently, for every closed set $F \subset Y, f^{-1}(F)$ is of multiplicative class $\alpha$ in $\left.X\right)$. The following properties of Borel mappings are well-known (see either [9] or [4]).

(2) $f$ is of class 0 iff $f$ is continuous.

(3) The maps of class $\alpha$ are also of class $\beta$ for all $\beta>\alpha$.

(4) If $f$ is of class $\alpha, g$ of class $\beta$ and $f \circ g$ is defined, then $f \circ g$ is of class $\alpha+\beta$.

Of particular importance to the present paper is the following theorem on limits of sequences of Borel mappings (see [4, p. 135]; cf. also [9, p. 386]).

(5) Let $X$ be a topological space, $Y$ a metric space, and let $f_{n}: X \rightarrow Y(n$ $=1,2, \cdots)$ be a sequence of mappings.

(i) If each $f_{n}$ is of class $\alpha$ and $f_{n} \rightarrow f$ uniformly, then $f$ is of class $\alpha$.

(ii) If each $f_{n}$ is of class $\alpha$ and $f_{n} \rightarrow f$ pointwise, then $f$ is of class $\alpha+1$.

3. Fully discrete families and $\sigma$-discrete mappings. Let $X$ be a topological space. A family $\mathscr{B}$ of subsets of $X$ is discrete (relative to $X$ ) if $X$ can be covered by open sets each having a nonvoid intersection with at most one member of $\mathfrak{B}$. The family $B$ will be called fully discrete if there exists a discrete collection $\left\{U_{B}\right\}$ of open sets in $X$ such that $B \subset U_{B}$ for each $B \in \mathscr{B}$. Spaces for which all discrete families are fully discrete are called collectionwise normal by Bing [3]. All paracompact Hausdorff (hence all metrisable) spaces have this property.

We will need the following lemma.

Lemma 2. Let $\left\{B_{d} \mid d \in D\right\}$ be a discrete family of sets of class $F_{\alpha}$ in a space $X$. Then $\cup B_{d}(d \in D)$ is also of class $F_{\alpha}$ in $X$.

The proof of Lemma 2 differs in no essential way from that of Lemma 1: The 
case when $\alpha=0$ is a familiar property of discrete families of closed sets (see e.g. [3]), and (for example) in the case when $\alpha=\beta+1$ we can replace the family $\left\{U_{d}\right\}$ of Lemma 1 with the family $\left\{\bar{B}_{d}\right\}$ of closures of the sets $B_{d}$ (recall that $\left\{\bar{B}_{d}\right\}$ is discrete whenever $\left\{B_{d}\right\}$ is [3]). The details are omitted.

Lemmas 1 and 2 combine to give us the following useful fact, the proof of which is clear.

Lemma 3. If $\left\{B_{d} \mid d \in D\right\}$ is a fully discrete family of sets of class $F_{\alpha}$ (respectively $\left.G_{\alpha}\right)$ in a space $X$, then $\cup B_{d}(d \in D)$ is of the same class in $X$.

A family $\mathscr{B}$ of subsets of a space $X$ is said to be a base for the mapping $f: X \rightarrow Y$ if, for every open set $G \subset Y, f^{-1}(G)$ is a union of sets from $\mathfrak{B}$. A mapping $f: X \rightarrow Y$ is said to be $\sigma$-discrete if it has a base $\mathscr{B}=\cup_{n=1}^{\infty} \mathscr{B}_{n}$ where each $\mathscr{B}_{n}$ is fully discrete (we will call such a family " $\sigma$-fully discrete").

The concept of a $\sigma$-discrete mapping was first introduced in [5] where we showed it plays a fundamental role in the theory of Borel mappings. A substantial part of the classical theory of Borel mappings, previously known only for separable metric spaces, was extended to general metric spaces in [5] for the class of $\sigma$-discrete mappings. And the present paper is essentially a continuation of that study. It is not difficult to see $[5, \S 3.2]$ that every mapping whose range has a countable base (hence every map with a separable metric range) is $\sigma$ discrete, and every continuous mapping with a metrisable range or domain is $\sigma$ discrete. Moreover, it was shown in [5, Theorem 3] that every Borel mapping defined on an analytic (hence any Borel) subset of a complete metric space into a metrisable space is $\sigma$-discrete. Hence the family of $\sigma$-discrete mappings constitute a rather extensive class of mappings. We now show that several of the basic properties of $\sigma$-discrete mappings proven in [5] for metric spaces, and which are fundamental for the results which follow, continue to hold for general topological spaces.

Proposition 1. The composite of two $\sigma$-discrete mappings is $\sigma$-discrete.

The proof in $[5, \S 3.2$ item (4)] can be used verbatim here; one needs only to replace the reference to collectionwise normality by the defining property of a fully discrete family.

Proposition 2. Let $X$ be a topological space, $Y$ a metrisable space and $f: X \rightarrow Y$ a mapping. Then a necessary and sufficient condition that $f$ be $\sigma$-discrete and of class $\alpha$ is that $f$ have a $\sigma$-fully discrete base of sets of additive class $\alpha$ in $X$.

Moreover for $\alpha>0$, we can replace "additive class $\alpha$ " by "ambiguous class $\alpha$ ". This continues to hold in the case $\alpha=0$ whenever $X$ is metrisable and 0-dimensional.

That the condition is sufficient follows from Lemma 3 and the properties of a base. To prove that the condition is necessary we can use the proof for the special case when $X$ is metrisable given in [5, Lemma 10 (3.4)] (see also Theorem 3 of [5]), since it depends only on the extension to general spaces of one basic fact. 
Namely, if $F \subset G \subset X$ where $F$ is of multiplicative class $\alpha>0$ and $G$ is of additive class $\alpha$, then there exists a set $K$ of ambiguous class $\alpha$ such that $F \subset K \subset G$. This is a special case of a general "separation theorem" which we prove below (Theorem 2, §4).

Proposition 3. Let $X, Y$, and $Z$ be topological spaces. Given mappings $f: X \rightarrow Y$ and $g: X \rightarrow Z$ which are $\sigma$-discrete, the "complex" mapping $h: X \rightarrow Y \times Z$, defined by $h(x)=(f(x), g(x))$ for $x \in X$, is also $\sigma$-discrete.

Proof. Let $\mathscr{B}=\cup_{n} \mathscr{B}_{n}$ and $\mathcal{C}=\cup_{m} \mathcal{C}_{m}$ be bases for $f$ and $g$ respectively, where $\mathscr{B}_{n}$ and $\mathcal{C}_{m}$ are fully discrete families for $n, m=1,2, \cdots$. Define $\mathbb{Q}_{n m}$ to be the family of all sets of the form $B \cap C$ where $B \in \mathscr{B}_{n}$ and $C \in \mathcal{C}_{m}$. It is easy to check that each $\mathbb{Q}_{n m}$ is fully discrete. Thus it suffices to show that each set of the form $h^{-1}(V \times W)$ is a union of sets from $\cup_{n, m} \mathbb{Q}_{n m}$, where $V$ and $W$ are open subsets of $Y$ and $Z$ respectively. Now $h^{-1}(V \times W)=f^{-1}(V) \cap g^{-1}(W)$, and $f^{-1}(V)=\cup_{n} \cup \mathfrak{B}_{n}^{\prime}$ and $g^{-1}(W)=\cup_{m} \cup \mathcal{C}_{m}^{\prime}$ for some $\mathfrak{B}_{n}^{\prime} \subset \mathfrak{B}_{n}$ and $\mathcal{C}_{m}^{\prime} \subset \mathcal{C}_{m}$, by the property of a base. Hence, if we let

$$
\mathbb{Q}_{n m}^{\prime}=\left\{B \cap C \mid B \in \mathscr{B}_{n}^{\prime} \text { and } C \in \mathcal{C}_{m}^{\prime}\right\}
$$

then we have $h^{-1}(V \times W)=\cup_{n, m} \mathbb{Q}_{n m}^{\prime}$, proving that $\cup_{n, m} \mathbb{Q}_{n m}$ is a $\sigma$-fully discrete base for $h$ (since the sets $V \times W$ are a base for the open sets of $Y \times Z$ ).

Corollary 1. If, in addition, $f$ and $g$ are of class $\alpha$, and both $Y$ and $Z$ are metrisable, then $h$ is of class $\alpha$.

Proof. For we may take, by Proposition 2 (since $Y \times Z$ is now metrisable), the members of $\mathscr{B}_{n}$ and $\mathcal{C}_{m}$ to be of additive class $\alpha$, and from this it follows that the members of $\mathbb{Q}_{n m}$ are of additive class $\alpha$. Since $\cup_{n, m} \mathbb{Q}_{n m}$ is a $\sigma$-fully discrete base for $h$, another application of Proposition 2 shows that $h$ is of class $\alpha$.

Remark. Note that if $f: X \rightarrow Y$ and $g: X \rightarrow Y$ are of class $\alpha$, where $Y$ is metrisable and $d$ is a metric for $Y$, and $r$ is some positive number, then

$$
\{x \in X \mid d(f(x), g(x)) \leq r\}
$$

is of multiplicative class $\alpha$ in $X$ by Corollary 1 and (2) and (3) of $\S 2$ (recalling that $d$ is a continuous mapping on $Y \times Y$ ).

4. A general reduction and separation theorem for Borel sets. In this section we prove generalizations of the standard "reduction" and "separation" theorems for sequences of Borel sets of additive and multiplicative classes (see [9, Theorems 1 and 2, p. 350]) A family $\mathfrak{B}$ of sets will be called a base for a family $\mathscr{E}$ if each member of $\mathcal{E}$ can be expressed as a union of members of $\mathscr{B}$.

Theorem 1 (Reduction theorem). Let $X$ be a topological space and $\left\{G_{a} \mid a \in I\right\}$ a collection of subsets of $X$ having a $\sigma$-fully discrete base of sets of ambiguous class $\alpha($ in $X)$. Then there exists a collection $\left\{H_{a} \mid a \in I\right\}$ of disjoint sets, having a $\sigma$-fully discrete base of sets of ambiguous class $\alpha$, such that 


$$
H_{a} \subset G_{a} \text { and } \bigcup_{a \in I} H_{a}=\bigcup_{a \in I} G_{a} \text {. }
$$

In addition, if $X=\cup_{a \in I} G_{a}$, then the sets $H_{a}$ are themselves of ambiguous class $\alpha$ in $X$.

Proof. By the hypothesis, there exist fully discrete collections $\left\{B_{n d} \mid d \in D_{n}\right\}(n$ $=1,2, \cdots)$ of sets of ambiguous class $\alpha$ in $X$ such that, for each $a \in I$, we have

$$
G_{a}=\bigcup_{n=1}^{\infty} \bigcup_{d \in D_{a n}} B_{n d}
$$

for suitable $D_{a n} \subset D_{n}$. We now define

$$
B_{n d}^{*}=B_{n d}-\bigcup_{m=1}^{n-1} \bigcup_{d \in D_{m}} B_{m d} .
$$

Since $\cup_{d \in D_{m}} B_{m d}$ is of ambiguous class $\alpha$ by Lemma 3 of $\S 3$, it follows that each $B_{n d}^{*}$ is of ambiguous class $\alpha$, since these sets form a field. Also, $B_{n d}^{*} \cap B_{m b}^{*}=\varnothing$ whenever $(n, d) \neq(m, b)$ (this is clear in the case when $n=m$ and $d \neq b$, since then $B_{n d} \cap B_{m b}=\varnothing$, and the other case follows immediately from the construction).

Now let $I$ be well ordered by some relation. We define, for each $a \in I$,

(3) $H_{a}=\cup_{n=1}^{\infty} \bigcup_{d \in D_{a n}}\left\{B_{n d}^{*} \mid a\right.$ is the least element of $I$ such that $\left.B_{n d}^{*} \subset G_{a}\right\}$.

The sets $H_{a}$ are disjoint, for $H_{a} \cap H_{a^{\prime}} \neq \varnothing$ implies $H_{a} \cap H_{a^{\prime}} \supset$ some $B_{n d}^{*}$, since the sets $B_{n d}^{*}$ are disjoint, and this can happen only if $a=a^{\prime}$ by (3). Moreover, since $B_{n d}^{*} \subset B_{n d}$, it is evident that the collection $\left\{B_{n d}^{*} \mid d \in D_{n}, n=1,2, \cdots\right\}$ is a (disjoint) $\sigma$-fully discrete base for $\left\{H_{a} \mid a \in I\right\}$.

It remains only to show that $\cup_{a \in I} G_{a} \subset \cup_{a \in I} H_{a}$, since $H_{a} \subset G_{a}$ is obvious from (3). Thus, let $x \in G_{a}$ be given and note that, by (1), there exists a minimal value for $n$ such that $x$ belongs to $\bigcup_{d \in D_{a n}} B_{n d}$. Consequently, $x$ belongs to some $B_{n d}^{*} \subset G_{a}$ by (2). Letting $b$ denote the least element in $I$ such that $B_{n d}^{*} \subset G_{b}$, it follows that $x \in H_{b}$. Hence $\cup_{a \in I} G_{a}=\cup_{a \in I} H_{a}$.

Since the collection $\left\{H_{a} \mid a \in I\right\}$ has a $\sigma$-fully discrete base of sets of ambiguous class $\alpha$, the union of every subcollection is of additive class $\alpha$. Thus, if $X=\cup_{a \in I} G_{a}$, then $H_{a}$ and $X-H_{a}=\cup H_{a^{\prime}}\left(a^{\prime} \in I, a^{\prime} \neq a\right)$ are both of additive class $\alpha$; consequently $H_{a}$ is of ambiguous class $\alpha$ in $X$.

This completes the proof of the theorem.

As in the standard case, we can use the above reduction theorem to deduce the following separation theorem.(Cf. [9, p. 350].)

Theorem 2 (Separation theorem). Let $X$ be a topological space, and let $\left\{F_{a} \mid a \in I\right\}$ be a collection of subset of $X$ such that $\bigcap_{a \in I} F_{a}=\varnothing$ and the collection $\left\{X-F_{a} \mid a \in I\right\}$ has a $\sigma$-fully discrete base of sets of ambiguous class $\alpha$. Then there exist sets $K_{a}(a \in I)$ of ambiguous class $\alpha$ in $X$ such that $K_{a} \supset F_{a}, \cap_{a \in I} K_{a}=\varnothing$, and $\left\{X-K_{a} \mid a \in I\right\}$ has a $\sigma$-fully discrete base of sets of ambiguous class $\alpha$.

In particular, if $F$ is of multiplicative class $\alpha$ and $G$ is of additive class $\alpha$ where 
$\alpha>0$ and $F \subset G$, then there exists a set $K$ of ambiguous class $\alpha$ such that $F \subset K \subset G$. This continues to hold in the case $\alpha=0$ provided $X$ is metrisable and 0-dimensional.

Proof. We apply the reduction theorem to the family of sets $G_{a}=X$ - $F_{a}(a \in I)$, and then put $K_{a}=X-H_{a}$ (in the notation of Theorem 1) for each $a \in I$. Also, since $\cup_{a \in I} G_{a}=X-\bigcap_{a \in I} F_{a}=X$, the sets $H_{a}$, and hence $K_{a}$, are of ambiguous class $\alpha$. Moreover, since we have $H_{a} \subset G_{a}$ and $\cup_{a \in I} H_{a}=\cup_{a \in I} G_{a}$ $=X$, and $X-K_{a}=H_{a}$, it follows that $K_{a} \supset F_{a}, \cap_{a \in I} K_{a}=\varnothing$, and the family $\left\{X-K_{a} \mid a \in I\right\}$ has a $\sigma$-fully discrete base of sets of ambiguous class $\alpha$.

To prove the particular case, assume $F$ and $G$ have the stated properties and observe that the sets $F$ and $X-G$ are then disjoint, and their complements $X-F$ and $G$, as sets of additive class $\alpha>0$, can each be written as a union of countably many sets of ambiguous class $\alpha$ (see [9, Theorem 1, p. 347]). Thus, $\{X-F, G\}$ has a $\sigma$-fully discrete base of sets of ambiguous class $\alpha$. If, in addition, $X$ is metrisable and 0 -dimensional, then the same is true in the case when $\alpha=0$, since $X$ will have a $\sigma$-discrete base (for the topology) of sets which are both open and closed (i.e., of ambiguous class 0 ). In either case, the above theorem applies and there exists a set $K$ of ambiguous class $\alpha$ in $X$ such that $K \supset F$ and $K \cap(X-G)=\varnothing$. The theorem is thus proved.

A basic result which we need for the proof of one of our main theorems is the following proposition on sets of ambiguous class $\alpha$. Its proof can be based (in part) on the above general separation theorem (cf. [9, Theorem 1, p 355]) or deduced directly from a general lemma of set theory (see [9, Lemma, p. 356]). In any case, the details can be found in $[9, \S 30$, IX].

Proposition 4. If $B$ is a set of ambiguous class $\alpha>1$ in a space $X$, then there exists a sequence of sets $B_{n}$ of ambiguous classes $<\alpha$ in $X$ such that

$$
B=\bigcup_{n=1}^{\infty}\left(B_{n} \cap B_{n+1} \cap \cdots\right)=\bigcap_{n=1}^{\infty}\left(B_{n} \cup B_{n+1} \cup \cdots\right) .
$$

Moreover, if $\alpha=\lambda+1$ where $\lambda$ is a limit ordinal, then the classes for the sets $B_{n}$ can be taken to be $<\lambda$.

Further, if $X$ is metrisable and 0-dimensional, then the result is also valid for $\alpha=1$.

5. Analytic representation of Borel mappings. The main results of this paper are proven in this section (Theorems 7 and 8 below). The preliminary theorems (Theorems 3 through 6) are essentially generalizations of Banach's key lemmas in [2], although they may also be of some independent interest.

Theorem 3. Let $X$ be a topological space, $Y$ a metric space, and let $f: X \rightarrow Y$ be $a$ Borel mapping of class $\alpha>0$. If, in addition, $f$ is $\sigma$-discrete then $f$ is the uniform limit of a sequence of $\sigma$-discrete mappings $f_{n}: X \rightarrow Y$, each of class $\alpha$, such that the 
range $f_{n}(X)$ is metrically discrete $(3)$ and contained in $f(X)(n=1,2, \cdots)$.

Furthermore, if $X$ is metrisable and 0-dimensional, then the theorem is also valid for $\alpha=0$.

Remark. In the case when $Y$ is separable (in which case the reference to " $\sigma$ discrete" mappings is superfluous) the theorem is known and due to Banach [2, Hilfssatz 4]. The present theorem answers a question of Kuratowski [11, p. 545] for the class of $\sigma$-discrete mappings, and thus whenever $X$ is an absolutely analytic metric space by $[5$, Theorem 3, p. 160].

Whether or not the reference to $\sigma$-discrete mappings is superfluous in general remains an open question.

Proof. For each $n=1,2, \cdots$, let $\left\{y_{n d} \mid d \in D_{n}\right\}$ be a maximal $1 / n$-discrete subset of $f(X)$; and set $V_{n d}=\left\{y \in Y \mid \rho\left(y, y_{n d}\right)<1 / n\right\}$, where $\rho$ is a fixed metric for $Y$. Then, evidently, $X=\bigcup\left\{f^{-1}\left(V_{n d}\right) \mid d \in D_{n}\right\}$ for each $n$. Since $f$ is $\sigma$ discrete and of class $\alpha$, it follows that $f$, and hence each of the families $\left\{f^{-1}\left(V_{n d}\right) \mid d \in D_{n}\right\}$, has a $\sigma$-fully discrete base of sets of ambiguous class $\alpha$ in $X$ by Proposition $2(\$ 3)$. By the reduction theorem (Theorem 1 ) there exist disjoint sets $H_{n d} \subset f^{-1}\left(V_{n d}\right)\left(d \in D_{n}\right)$ such that $\left\{H_{n d} \mid d \in D_{n}\right\}$ has a $\sigma$-fully discrete base of sets of ambiguous class $\alpha$. Hence, for each $n=1,2, \cdots$, we can define a mapping $f_{n}: X \rightarrow f(X)$ by the rule $f_{n}(x)=y_{n d}$ iff $x \in H_{n d}$. The properties of the sets $H_{n d}$ just mentioned, when translated into properties of $f_{n}$, say that $f_{n}$ is well defined, $\sigma$-discrete and of class $\alpha$. Moreover, $f_{n}(X)\left(=\left\{y_{n d} \mid d \in D_{n}\right\}\right)$ is $1 / n$ discrete and contained in $f(X)$.

It remains only to show that $f$ is the uniform limit of the sequence $\left\{f_{n}\right\}$. But, for any $x \in X$, we have $\rho\left(f(x), f_{n}(x)\right)<1 / n$, since $f_{n}(x)=y_{n d}$ implies $x \in H_{n d}$ $\subset f^{-1}\left(V_{n d}\right)$; i.e., that $\rho\left(f(x), y_{n d}\right)<1 / n$. It follows that the convergence is uniform, and this completes the proof of the theorem.

We now show that the mappings $f_{n}$ in the above theorem are themselves pointwise limits of sequences of $\sigma$-discrete Borel mappings of lower class.

Theorem 4. Let $X$ be a topological space, $Y$ a metric space, and let $f: X \rightarrow Y$ be a Borel mapping of class $\alpha>1$ such that the range of $f$ is discrete. If, in addition, $f$ is $\sigma$-discrete, then $f$ is the (pointwise) limit of a sequence of $\sigma$-discrete mappings $f_{n}: X \rightarrow Y$, each of class $<\alpha$ and taking its values in $f(X)$. Also, if $\alpha=\lambda+1$, where $\lambda$ is a limit ordinal, then these mappings can be made to be of classes $<\lambda$.

Further, if $X$ is metrisable and 0-dimensional, then the theorem is also valid for $\alpha=1$.

Remark. In the case when $Y$ is separable, the theorem is due to Banach [2, Hilfssatz 5], and our proof is to a large extent a generalization of his.

Whether or not the theorem remains true without the reference to $\sigma$-discrete mappings is unknown.

Proof. Let $f(X)=\left\{y_{d} \mid d \in D\right\}$ and note that the collection $\left\{f^{-1}\left(y_{d}\right) \mid d\right.$

(3) A subset $E$ of $Y$ is said to be metrically discrete, relative to a given metric $\rho$ for $Y$, if for some $\varepsilon>0$ we have $\rho(x, y) \geq \varepsilon$ for each pair of distinct points $x, y \in E$ (in which case we say that $E$ is $\varepsilon$-discrete). 
$\in D\}$ has a $\sigma$-fully discrete base $\mathscr{B}=\cup \mathscr{B}_{m}(m=1,2, \cdots)$, where each $\mathscr{B}_{m}$ is a fully discrete collection of sets of ambiguous class $\alpha$ (by Proposition 2). It follows that each $B_{m}=\bigcup\left\{B \mid B \in \mathscr{B}_{m}\right.$ and $B \subset$ some $\left.f^{-1}\left(y_{d}\right)\right\}$ is of ambiguous class $\alpha$ in $X$ (by Lemma 3), and that $X=\cup_{m=1}^{\infty} B_{m}$. Hence, by Proposition 4, there exist sequences of sets $B_{m}^{n}(n=1,2, \cdots)$ each of some ambiguous class $\alpha_{n m}<\alpha$ [resp. $<\lambda$, if $\alpha=\lambda+1$ ] such that

$$
B_{m}=\bigcup_{n=1}^{\infty}\left(B_{m}^{n} \cap B_{m}^{n+1} \cap \cdots\right)=\bigcap_{n=1}^{\infty}\left(B_{m}^{n} \cup B_{m}^{n+1} \cup \cdots\right) .
$$

Also, since $\mathscr{B}_{m}$ is fully discrete, we can find open sets $U_{B} \supset B$ such that the collection $\left\{U_{B} \mid B \in \mathscr{B}_{m}\right\}$ is discrete for each $m=1,2, \cdots$.

Now, given $x \in X=\cup_{m=1}^{\infty} B_{m}$, there exists some $m$ such that $x \in B_{m}$ and $x \notin B_{p}$ for $p=1, \cdots, m-1$. Hence, by (1), there exists an index $n(m)$ such that $x \in B_{m}^{n(m)} \cap B_{m}^{n(m)+1} \cap \cdots$, and also indices $n(p)$, for $p=1, \cdots, m-1$, such that $x \notin B_{p}^{n(p)} \cup B_{p}^{n(p)+1} \cup \cdots$. Thus, for all $n \geq n(1), n(2), \cdots, n(m)$, we have

$$
x \in B_{m}^{n}-\bigcup_{p=1}^{m-1} B_{p}^{n} .
$$

With this in mind, we select a point $y_{0} \in f(X)$ arbitrarily and define mappings $f_{n}: X \rightarrow f(X)(n>1)$ as follows:

Case 1. If $x$ belongs to $B_{m}^{n}-\cup_{p=1}^{m-1} B_{p}^{n}$ for some $n>m \geq 1$, then define $f_{n}(x)=y_{d}$ iff for some $B \in \mathscr{B}_{m}, x \in U_{B}$ and $\varnothing \neq B \subset f^{-1}\left(y_{d}\right)$. Otherwise, define $f_{n}(x)=y_{0}$.

Case 2. If $x$ belongs to $X-\cup_{p=1}^{n-1} B_{p}^{n}$, then define $f_{n}(x)=y_{0}$.

The mappings $f_{n}$ are well defined in Case 1, since the sets $U_{B}$ are disjoint for $B \in \mathscr{B}_{m}$ and $\varnothing \neq B \subset f^{-1}\left(y_{d}\right)$ for at most one $d \in D$. Moreover, by (2) above, given $x \in X$ there exists an $m$ and $N>m$ such that $x \in B_{m}^{n}-\bigcup_{p=1}^{m-1} B_{p}^{n}$ for all $n \geq N$; and (since $x \in B_{m}$ by (1)) there exists a $B \in \mathscr{B}_{m}$ such that $x \in B$ $\subset f^{-1}\left(y_{d}\right)$ for some $d \in D$. Consequently, $f_{n}(x)=y_{d}=f(x)$ for every $n \geq N$, and so the sequence $f_{n}$ converges pointwise to $f$. Thus it remains only to show that each $f_{n}$ is $\sigma$-discrete and of class $<\alpha$. But the collections $\left\{U_{B} \cap\left(B_{m}^{n}-\cup_{p=1}^{m-1} B_{p}^{n}\right)\right\}$ $\left.B \notin \mathscr{B}_{m}\right\}$, for $m=1,2, \cdots, n-1$, and $\left\{X-\cup_{p=1}^{n-1} B_{p}^{n}\right\}$ are evidently fully discrete, and hence constitute a $\sigma$-fully discrete base for $f_{n}(n=2,3, \cdots)$ as can easily be seen from the defining conditions for this mapping and the fact that $\left\{y_{d} \mid d \in D\right\}$ is discrete. Moreover, since $B_{m}^{n}$ is of ambiguous class $\alpha_{m n}<\alpha$, the sets making up this base for $f_{n}$ are all of ambiguous class (additive class in the case when $\alpha=1) \alpha_{n}=\max \left\{\alpha_{n m} \mid m=1,2, \cdots, n-1\right\}<\alpha$ [resp. $<\lambda$, if $\alpha$ $=\lambda+1$ ]. It follows by Proposition 2 that $f_{n}$ is of class $<\alpha$.

This completes the proof of the theorem.

We now prove a lemma which paves the way for showing that the "double sequence" obtained when Theorems 3 and 4 are combined, can be reduced to a single sequence having the required properties. 
Lemma 4. Let $X$ be a topological space, $Y$ a metric space, and $f_{n}, g_{n}: X$ $\rightarrow Y(n=1,2, \cdots)$ two convergent sequences of $\sigma$-discrete mappings of classes $<\alpha$, each having a discrete range, with pointwise limits $f$ and $g$ respectively. Further, suppose $c$ is a number satisfying $\rho(f(x), g(x))<c$ for all $x$ in $X$, where $\rho$ is a metric for $Y$. Then there exists a sequence of $\sigma$-discrete mappings $h_{n}: X \rightarrow Y$ of classes $<\alpha$, each having a discrete range $\subset f_{n}(X) \cup g_{n}(X)$, such that $g$ is the pointwise limit of $\left\{h_{n}\right\}$ and $\rho\left(h_{n}(x), f_{n}(x)\right) \leq c$ for all $x$ in $X$. (Cf. [2, Hilfssatz 6] and [9, Theorem 5, p. 389].)

Proof. Let $d_{n}: X \rightarrow Y \times Y$ be the complex mapping with coordinates $f_{n}$ and $g_{n}$, and let $\rho_{n}=\rho \circ d_{n}$; hence, $\rho_{n}(x)=\rho\left(f_{n}(x), g_{n}(x)\right)$ for each $x \in X$. Since $d_{n}$ is $\sigma$ discrete and of class $<\alpha(\S 3$, Proposition 3) and $\rho$ is continuous on $Y \times Y$, it follows that $\rho_{n}$ is $\sigma$-discrete and of class $<\alpha$ (Proposition 1 and (4) of $\S 2$ ). Therefore, since $f_{n}(X) \times g_{n}(X)$ is discrete, which follows easily from the hypothesis, the set

$$
A_{n}=\left\{x \in X \mid \rho\left(f_{n}(x), g_{n}(x)\right) \leq c\right\}
$$

is of ambiguous class $<\alpha$ in $X$, as the inverse image under $d_{n}$ of a subset of $f_{n}(X) \times g_{n}(X)$. Accordingly, we define $h_{n}(x)=g_{n}(x)$ if $x$ belongs to $A_{n}$, and $h_{n}(x)=f_{n}(x)$ otherwise. Since $A_{n}$ is of ambiguous class $<\alpha$ and $g_{n} \mid A_{n}$ and $f_{n} \mid X-A_{n}$ are both of class $<\alpha$, it follows easily that $h_{n}$ is of class $<\alpha$. Moreover, it is clear that $h_{n}$ is $\sigma$-discrete (by intersecting a $\sigma$-discrete base for $g_{n}$ and $f_{n}$ with the sets $A_{n}$ and $X-A_{n}$ respectively), and that $\rho\left(h_{n}(x), f_{n}(x)\right) \leq c$ for each $x$ in $X$. Finally, for a given $x \in X$, we may choose an $m$ sufficiently large so that $\rho\left(g_{n}(x), f_{n}(x)\right) \leq c$ for all $n \geq m$; that is, such that $h_{n}(x)=g_{n}(x)$ for all $n \geq m$. It follows that $\lim h_{n}(x)=\lim g_{n}(x)=g(x)$, thus completing the proof of the lemma.

We now show that the $\sigma$-discrete Borel mappings of class $\alpha>1$ are members of the "Banach" class $\Phi_{\alpha}^{*}$ (respectively $\Phi_{\alpha-1}^{*}$ ) whenever $\alpha$ is a finite or limit ordinal (respectively an infinite nonlimit ordinal).

Theorem 5. Let $X$ be a topological space, $Y$ a metric space, and let $f: X \rightarrow Y$ be a Borel mapping of class $\alpha>1$ which is $\sigma$-discrete. Then $f$ is the (pointwise) limit of a sequence of $\sigma$-discrete mappings $f_{n}: X \rightarrow Y$ of classes $<\alpha$, such that each has $a$ discrete range contained in the range of $f$. Further, if $\alpha=\lambda+1$, where $\lambda$ is a limit ordinal, then these mappings can be taken to be of classes $<\lambda$.

Moreover, the theorem is also valid in the case $\alpha=1$ whenever Theorem 4 holds for $\alpha=1$; hence, in particular, when $X$ is metrisable and 0-dimensional.

Remark. We remark that the proof for the case when $Y$ is separable can now be used verbatim (see e.g. [9, p. 390]), since it depends only on the preceding generalizations. However, we include it here for the sake of completeness.

Proof. By Theorem $3, f$ is the uniform limit of a sequence $\left\{f_{m}\right\}$ of $\sigma$-discrete mappings of class $\alpha$ each with a discrete range $\subset f(X)$; and we may assume that 


$$
\rho\left(f_{m+1}(x), f_{m}(x)\right)<2^{-m}
$$

for each $x \in X$ (by replacing, if necessary, the given sequence by a suitable subsequence). By Theorem 4, each $f_{m}$ is the pointwise limit of a sequence $\left\{f_{m n}\right\}$ of $\sigma$-discrete mappings of classes $<\alpha$ [resp. $<\lambda$ ] each having a discrete range $\subset f_{m}(X)$.

By induction on $m$ we now define a double sequence of $\sigma$-discrete mappings $h_{m n}$ each of class $<\alpha$ [resp. $\left.<\lambda\right]$ and having a discrete range $\subset f_{m n}(X)$ $\cup f_{m+1, n}(X) \subset f(X)$, such that $\lim h_{m n}(x)=f_{m}(x)$ and $\rho\left(h_{m+1, n}(x), h_{m n}(x)\right) \leq 2^{-m}$ for all $x$ in $X$.

We take $h_{1 n}=f_{1 n}$ for $n=1,2, \cdots$. Then, assuming the sequence $\left\{h_{m n}\right\}$ has been defined with the desired properties, we obtain the $m+1$ sequence by applying the preceding lemma to the convergent sequences $h_{m n} \rightarrow f_{m}$ and $f_{m+1, n} \rightarrow f_{m+1}$ (in the roles of $f_{n} \rightarrow f$ and $g_{n} \rightarrow g$, respectively), and with $c=2^{-m}$ (in view of (1)). The induction is then complete, and the proof will be complete if we can show that $f$ is the pointwise limit of $\left\{h_{n n}\right\}$.

Given $x$ in $X$ and $\varepsilon>0$, we can choose $m$ so that $2^{-m+1}<\varepsilon$ and $\rho\left(f_{m}(x), f(x)\right)$ $<\varepsilon$. Accordingly, we can find $N>m$ such that $\rho\left(h_{m n}(x), f_{m}(x)\right)<\varepsilon$ whenever $n>N$. It follows that for $n>N$ we have

$$
\begin{aligned}
\rho\left(h_{n n}(x), f(x)\right) \leq & \rho\left(h_{n n}(x), h_{n-1, n}(x)\right)+\cdots+\rho\left(h_{m+1, n}(x), h_{m n}(x)\right) \\
& +\rho\left(h_{m n}(x), f_{m}(x)\right)+\rho\left(f_{m}(x), f(x)\right) \\
\leq & \left(2^{-n+1}+\cdots+2^{-m}\right)+\varepsilon+\varepsilon<3 \varepsilon,
\end{aligned}
$$

proving the desired convergence.

The only case left to treat to obtain an analogue of Theorem 5 for the Baire classes $\Phi_{\alpha}$ is the case when $\alpha=1$ and $Y$ has the extension property for $X$ (see $\S 1)$. We now prove this important theorem.

Theorem 6. Let $X$ be a topological space, $Y$ a metric space having the extension property for $X$, and let $f: X \rightarrow Y$ be a Borel mapping of class 1 . If, in addition, $f$ is $\sigma$-discrete, then $f$ is the limit of a sequence of continuous mappings from $X$ to $Y$.

Proof. In view of the second part of Theorem 5, it is enough to prove the present theorem under the additional hypothesis that $f(X)$ is discrete. Let $f(X)=\left\{y_{d} \mid d \in D\right\}$, and let $\mathscr{B}=\cup_{m} \mathscr{B}_{m}$ be a base for $f$, where $\mathscr{B}_{m}$ is a fully discrete collection of $F_{\sigma}$ sets in $X$ for each $m$ (the existence of such a base being a consequence of Proposition 2, §3). For each $B$ in $\mathscr{B}$ we write $B=\cup_{n=1}^{\infty} F_{B n}$ where $F_{B n}$ is closed in $X$ and $F_{B n} \subset F_{B n+1}$ for each $n$. Then, for each $m=1,2, \cdots$, we let $F_{m n}=\bigcup\left\{F_{B n} \mid B \in \mathscr{B}_{m}\right.$, and $B \subset$ some $\left.f^{-1}\left(y_{d}\right)\right\}$. Since $\mathfrak{B}_{m}$ is fully discrete, it follows that each $F_{m n}$ is closed in $X$ by Lemma 3; and we have $F_{m n} \subset F_{m n+1}$ for each $n$. Moreover, since the sets $f^{-1}\left(y_{d}\right)$ are open and cover $X$, we have $X=\bigcup F_{m n}(m, n=1,2, \cdots)$ by the properties of a base.

We now construct continuous mappings $f_{n}: \cup_{m=1}^{n} F_{m n} \rightarrow Y(n=1,2, \cdots)$ by 
defining $f_{n}(x)=y_{d}$ iff $x \in F_{B n} \subset f^{-1}\left(y_{d}\right)$ for some $B \in \cup_{m=1}^{n} \mathscr{B}_{m}$. Continuity follows from the fact that the collections $\left\{F_{B n} \mid B \in \mathscr{B}_{m}\right.$ and $B \subset$ some $\left.f^{-1}\left(y_{d}\right)\right\}$, for $m=1,2, \cdots, n$, form a locally-finite base of closed sets for $f_{n}$; and hence the inverse image of every set under $f_{n}$ is closed.

By hypothesis, each $f_{n}$ has a continuous (hence also $\sigma$-discrete) extension $f_{n}^{*}$ to all of $X$. The proof will be complete if we can show that $f(x)=\lim f_{n}^{*}(x)$ for each $x$ in $X$. But, given $x \in X$, there exists $m_{0}$ and $n_{0}$ such that $x \in F_{m_{0} n_{0}}$, and so $x \in F_{m_{0} n}$ for all $n \geq n_{0}$, since the sequence $\left\{F_{m_{0} n}\right\}$ is increasing. Consequently, for all $n>$ both $m_{0}$ and $n_{0}$ we have $x \in \cup_{m=1}^{n} F_{m n}$, and thus $f_{n}^{*}(x)=f_{n}(x)=f(x)$; hence $f(x)=\lim f_{n}^{*}(x)$. This completes the proof of the theorem.

Remark. Theorem 6 provides an alternate proof of Theorem 5 in the case when $X$ is metrisable and 0-dimensional. For, by a theorem of Stone [12, Theorem 3', p. 8], every closed subset of $X$ is a retract of $X$, so any $Y$ would have the extension property for $X$.

We are now in a position to give our main theorems, which are generalizations of the Banach and Lebesgue-Hausdorff theorems cited in the introduction of this paper. We begin with the generalization of Banach's theorem [2].

Theorem 7. Let $X$ be a topological space and $Y$ a metric space, and let $\Sigma$ denote the family of all $\sigma$-discrete mappings from $X$ to $Y$. Then the family $\Sigma \cap \Phi_{\alpha}^{*}$ coincides with the family of all $\sigma$-discrete Borel mappings of class $\alpha$, respectively $\alpha+1$, according as $\alpha$ is finite or infinite $\left(1 \leq \alpha<\omega_{1}\right)$.

In particular, if $X$ is an absolutely analytic metric space, then the theorem is true with the references to $\sigma$-discrete mappings omitted.

Proof. We have already noted (see $\$ 1$ and (5) of $\$ 2$ ) that each member of $\Sigma \cap \Phi_{\alpha}^{*}$ is a $\sigma$-discrete Borel mapping of class $\alpha$, respectively $\alpha+1$, according as $\alpha$ is finite or infinite. On the other hand, the $\sigma$-discrete Borel mappings of class 1 from $X$ to $Y$ are, by definition, the members of $\Sigma \cap \Phi_{1}^{*}$. Thus, proceeding inductively, suppose we have shown that every $\sigma$-discrete Borel mapping of class $\beta$, respectively of class $\beta+1$, belongs to the family $\Sigma \cap \Phi_{\beta}^{*}$ for all finite ordinals, respectively infinite ordinals, $\beta<\alpha$. There are two cases to consider:

(i) $\alpha=\lambda$, where $\lambda$ is a limit ordinal. Let $f$ be a $\sigma$-discrete Borel map of class $\lambda+1$. Then, by Theorem $5, f$ is the limit of a sequence of $\sigma$-discrete mappings $f_{n}$ of classes $\beta_{n}<\lambda$; and we may assume that each $\beta_{n}$ is a nonlimit ordinal. By the inductive hypothesis, each $f_{n}$ belongs to $\Sigma \cap \Phi_{\beta_{n}}^{*}(n=1,2, \cdots)$, and so $f$ belongs to $\Sigma \cap \Phi_{\lambda}^{*}$ (by definition).

(ii) $\alpha=\lambda+m$, where $\lambda$ is a limit ordinal (resp. $\lambda=0$ ) and $m=1,2, \cdots$ (resp. $m=2,3, \cdots$ ). If $f$ is a $\sigma$-discrete Borel mapping of class $\lambda+m+1$ (resp. $m$ ), then, again by Theorem $5, f$ is the limit of a sequence of $\sigma$-discrete mappings $f_{n}$ of class $\lambda+m$ (resp. $m-1$ ). Again, by the inductive hypothesis, each $f_{n} \in \Sigma \cap \Phi_{\lambda+m-1}^{*}$ and so $f \in \Sigma \cap \Phi_{\lambda+m}^{*}(\lambda$ a limit ordinal or $\lambda=0)$.

All that remains is to check the particular case when $X$ is an absolutely analytic metric space. But this follows immediately upon observing that, for such spaces $X$, all Borel mappings are $\sigma$-discrete [5, Theorem 3]. 
We now can prove the following generalized Lebesgue-Hausdorff theorem (cf. $\S 1)$.

Theorem 8. Let $X$ be a topological space and $Y$ a metric space having the extension property for $X(\S 1)$, and let $\Sigma$ denote the family of all $\sigma$-discrete mappings from $X$ to $Y$. Then the family $\Sigma \cap \Phi_{\alpha}$ coincides with the family of all $\sigma$-discrete Borel mappings of class $\alpha$, respectively $\alpha+1$, according as $\alpha$ is finite or infinite.

In particular, if $X$ is an absolutely analytic metric space and either $X$ is metrisable and 0-dimensional or $Y$ is a locally convex topological linear space, then the above is true without any reference to $\sigma$-discrete mappings or the extension property.

Proof. We have already remarked ( $\$ 1$ and (5) of $\S 2)$ that the class $\Sigma \cap \Phi_{\alpha}$ is contained in the family of all $\sigma$-discrete Borel mappings of class $\alpha$ or $\alpha+1$, depending on whether $\alpha$ is finite or infinite, respectively.

Conversely, for $\alpha=1$, the containment of the family of Borel mappings of class 1 in the class $\Sigma \cap \Phi_{1}$ is precisely the content of Theorem 6. Thus, it follows that $\Sigma \cap \Phi_{1}=\Sigma \cap \Phi_{1}^{*}$, and hence that $\Sigma \cap \Phi_{\alpha}=\Sigma \cap \Phi_{\alpha}^{*}$ for $1 \leq \alpha<\omega_{1}$ by the definition of these classes. The theorem now follows from Theorem 7 .

The particular cases follow from the fact that whenever these conditions are satisfied $Y$ has the extension property for $X$ (see [8, p. 57] and [10, p. 351]) and all Borel mappings are $\sigma$-discrete [5, Theorem 3].

6. Extending Borel mappings. We apply the results of the preceding section to prove some basic theorems on the extension properties of Borel mappings.

Theorem 9. Let $X$ be a paracompact space, $Y$ a complete metric space, and $f: A \subset X \rightarrow Y$ a $\sigma$-discrete Borel mapping of class $\alpha$, for some nonlimit ordinal $\alpha$. Then $f$ can be extended to a Borel mapping $f^{*}: A^{*} \subset X \rightarrow Y$ of class $\alpha$ such that $A^{*}$ is of multiplicative class $\alpha+1$ in $X\left(A \subset A^{*}\right)$.

Proof. The proof will be by transfinite induction on the class of the mapping. For $\alpha=0$, the theorem is a well-known property of continuous mappings [9, $\mathrm{p}$. 422]. Hence let $\alpha=\beta+1$ be a countable ordinal $>0$ and suppose the theorem is true for all (nonlimit) ordinals $<\alpha$. Let $d$ be a complete metric for $Y$, and let $h$ be an isometric embedding of $(Y, d)$ into the Banach space $\left(Y^{*}, d^{*}\right)$ of all bounded continuous real-valued functions on $Y$, where $d^{*}$ is the metric derived from the sup-norm. (See [8, pp. 63-64].) By a theorem of Arens [1], the Banach space $Y^{*}$ has the extension property for the class of all paracompact (= fully normal) spaces. Thus, by Theorems 5 and 6 , the composite mapping $h \circ f$ (which is $\sigma$-discrete and of class $\alpha$ ) is the limit of a sequence $\left\{f_{n}\right\}$ of $\sigma$-discrete mappings of class $\beta$. Now, by the induction hypothesis, each $f_{n}$ can be extended to a mapping $f_{n}^{*}: A_{n}^{*} \subset X \rightarrow Y^{*}$ of class $\beta$, where $A_{n}^{*}$ contains $A$ and is of multiplicative class $\beta+1=\alpha$ in $X$. We now put $A^{\prime}=\cap A_{n}^{*}(n=1,2, \cdots)$, and denote by $C_{n p k}$ the set of all $x \in A^{\prime}$ such that

$$
d^{*}\left(f_{n}^{*}(x), f_{n+p}^{*}(x)\right) \leq 1 / k \quad(n, p, \text { and } k=1,2, \cdots) .
$$


It follows (cf. the proof of Lemma 4 in $\S 5$ ) that $C_{n p k}$ is of multiplicative class $\beta$ in $A^{\prime}$. Hence the set

$$
A^{*}=\bigcap_{k=1}^{\infty} \bigcup_{n=1}^{\infty} \bigcap_{p=1}^{\infty} C_{n p k}
$$

is of multiplicative class $\beta+2=\alpha+1$ in $A^{\prime}$. Moreover, since it is clear that $A^{\prime}$ is of multiplicative class $\alpha$ in $X$, it follows that $A^{*}$ is of multiplicative class $\alpha+1$ in $X$. We show that $A^{*}$ has the properties ascribed to it in the statement of the theorem.

Observe first that $x \in A^{*}$ if and only if the sequence $\left\{f_{n}^{*}(x)\right\}$ is Cauchy in $Y^{*}$, by the construction of $A^{*}$, and hence $\left\{f_{n}^{*}(x)\right\}$ converges to some point $f^{\circ}(x)$ $\in Y^{*}$; i.e., $F^{o}$ is the pointwise limit of the sequence $\left\{f_{n}^{*}\right\}$ on $A^{*}$. It follows from (5) of $\S 2$ that $f^{o}: A^{*} \rightarrow Y^{*}$ is a Borel mapping of class $\beta+1=\alpha$. Also, from the fact that $f_{n} \rightarrow h \circ f$ on $A$, we have $A \subset A^{*}$ and $f^{0} \mid A=h \circ f$.

To complete the proof we need to have $f^{\circ}\left(A^{*}\right) \subset h(Y)$, which may not be the case at present. However, since $Y$ is isometrically embedded in $Y^{*}, h(Y)$ is complete and hence closed in $Y^{*}$. Consequently, $f^{o-1}(h(Y))$ is of multiplicative class $\alpha$ in $A^{*}$. Hence we may assume without loss that $A \subset A^{*} \subset f^{o-1}(h(Y))$. It now follows that $f^{*}=h^{-1} \circ f^{o}: A^{*} \rightarrow Y$ is of class $\alpha$ and extends $f$ as required.

Remark. In the case when $X$ is metrisable and $Y$ is separable metric, Theorem 9 is known and due to Kuratowski [12]; where the theorem includes also the case when $\alpha$ is a limit ordinal $\left(<\omega_{1}\right)$. It is interesting to note that Kuratowski's proof does not use transfinite induction (see [9, p. 434]), and this may account for his inclusion (and our exclusion) of the case for limit ordinals. (Of course, our Theorem 9 shows that a map of class $\lambda$, for some limit ordinal $\lambda<\omega_{1}$, does extend to a map of class $\lambda+1$ defined on a set of multiplicative class $\lambda+2$; but whether or not these ordinals can be sharpened to $\lambda$ and $\lambda+1$, respectively, is an open question.) What seems to be lacking in using transfinite induction, in the case of a limit ordinal $\lambda$, is a representation of maps of class $\lambda$ as limits of maps of lower classes where the mode of convergence is stronger than that of pointwise convergence. (We recall that the pointwise limit of a sequence of mappings of classes $<\lambda$ may be of class $\lambda+1$ and not of class $\lambda$ by Theorem 7.) Now the uniform limit of a sequence of mappings of classes $\langle\lambda$ is a map of class $\lambda$, but the converse is definitely not true (even for $\sigma$-discrete mappings); which is contrary to what was thought to be the case in [6] and the subsequent reference to [6] in [14] (Theorem 16 on p. 64 of [6] is false). For example, if $B \subset X$ is of exact ambiguous class $\lambda$ in $X$ and $f: X \rightarrow\{0,1\}$ is the characteristic function for $B$, then $f$ is of class $\lambda$ but the only sequences of mappings which converge uniformly to $f$ (and take their values in $\{0,1\}$ ) are those which are eventually equal to $f$.

A further remark in connection with Theorem 9 relates to the question of whether or not the extended mapping $f^{*}$ is $\sigma$-discrete. In the case when $X$ is metrisable the answer is affirmative, since we may first extend to $A^{*} \subset \tilde{X}$ (where 
$\tilde{X}$ is the metric completion of $X$ ) and then restrict to $A^{*} \cap X$; the mapping will then be $\sigma$-discrete by [5, Theorem 3 and §3.1]. The mapping will always be $\sigma$ discrete if it is true that the pointwise limit of a sequence of $\sigma$-discrete mappings is $\sigma$-discrete, but this too remains an open question.

Recall that if $f$ is a bijection such that $f$ is of class $\alpha$ and $f^{-1}$ is of class $\beta$, then $f$ is said to be a generalized homeomorphism of class $(\alpha, \beta)[9$, p. 374]. For $\alpha=\beta=0$ this reduces to the usual notion of a homeomorphism. If both $f$ and $f^{-1}$ are $\sigma$-discrete, we will say that $f$ is bi- $\sigma$-discrete.

Theorem 9 enables us to prove the following generalization of the well-known Lavrentiev theorem on extending homeomorphisms [9, p. 429].

Theorem 10. Let $\alpha$ and $\beta$ be two given nonlimit countable ordinals. Then any bi- $\sigma-$ discrete generalized homeomorphism of class $(\alpha, \beta)$, between arbitrary subsets $A$ and $B$ of complete metric spaces $X$ and $Y$, can be extended to two subsets of classes $\alpha+\beta+1$ and $\beta+\alpha+1$, respectively, such that the extension is also bi- $\sigma$-discrete and of class $(\alpha, \beta)$.

Remark. For $\alpha=\beta=0$ the theorem is well-known and due to Lavrentiev [9, p. 429]. For higher classes in the case when $X$ and $Y$ are separable, the theorem is due to Kuratowski [12], and our proof is a modification of his (cf. [9, p. 436]).

Proof. Let the mapping $f$ satisfy the hypothesis for the spaces mentioned. Then, by the preceding theorem, there exists an extension $f^{*}$ of $f$, of class $\alpha$, to a set $A^{*}$ of multiplicative class $\alpha+1$ in $X$. Put $I=\left\{(x, y) \mid y=f^{*}(x)\right\}$. Now let $B^{*}, g^{*}$, and $I$ have the same meaning with respect to $g=f^{-1}$. By $[5$, Corollary to Theorem 4, p. 163], $I$ and $J$ are of multiplicative classes $\alpha+1$ and $\beta+1$, respectively, in $X \times Y$. By Corollary $1(\S 3)$, the complex mapping $h: A^{*} \rightarrow I$ defined by $h(x)=\left(x, f^{*}(x)\right)$ is of class $\alpha$, and so

$$
h^{-1}(J)=h^{-1}(I \cap J)=\left\{x \in A^{*} \mid\left(x, f^{*}(x)\right)=\left(g^{*}(y), y\right) \text { for some } y \in B^{*}\right\}
$$

is of multiplicative class $\alpha+\beta+1$ [9, Theorem 1, p. 376]. Similarly, we define the complex mapping $k: B^{*} \rightarrow J$ by $k(y)=\left(g^{*}(y), y\right)$, and note that $k^{-1}(I)$ $=k^{-1}(I \cap J)$ is of multiplicative class $\beta+\alpha+1$. One now easily verifies that $f^{*} \mid h^{-1}(J)$ is a generalized homeomorphism of class $(\alpha, \beta)$ onto $k^{-1}(I)$. Finally, it is bi- $\sigma$-discrete since it is defined between two absolute Borel spaces [5, Theorem 3, p. 160].

Our final theorem answers a question of A. H. Stone [13, 7.1(b), p. 29] in the case of a bi- $\sigma$-discrete generalized homeomorphism.

Theorem 11. If $A$ is absolutely Borel of multiplicative class $\alpha>0$ and $f: A \rightarrow Y$ is a generalized homeomorphism of class $(\alpha, \beta)$ such that either $f^{-1}$ is $\sigma$-discrete or $f(A)$ is absolutely analytic, then $f(A)$ is absolutely Borel of multiplicative class $\beta+\alpha$ ( $\alpha$ and $\beta$ are assumed to be nonlimit ordinals).

Proof. The hypothesis ensures that $f$ is bi- $\sigma$-discrete [5, Theorem 3]. We may, of course, assume that $Y$ is complete. Now, if in the proof of the preceding 
theorem we let $A^{*}=A$ and $f^{*}=f$, then one sees without difficulty that $h^{-1}(J)=A$ and $k^{-1}(I)=f(A)$. Since $k^{-1}(I)$ is of multiplicative class $\beta+\alpha$ with respect to $B^{*}$ and $B^{*}$ is of multiplicative class $\beta+1$ in $Y$, it follows that $f(A)$ is absolutely Borel of multiplicative class $\beta+\alpha$.

\section{REFERENCES}

1. R. Arens, Extensions of functions on fully normal spaces, Pacific J. Math. 2 (1952), 11-22. MR 14, 191.

2. S. Banach, Über analytisch darstellbare Operationen in abstrakten Räumen, Fund. Math. 17 (1931), 283-295.

3. R. H. Bing, Metrization of topological spaces, Canad. J. Math. 3 (1951), 175-186. MR 13, 264.

4. G. Choquet, Lectures on analysis. Vol. I: Integration and topological vector spaces, Benjamin, New York, 1969. MR 40 \#3252.

5. R. W. Hansell, Borel measurable mappings for nonseparable metric spaces, Trans. Amer. Math. Soc. 161 (1971), 145-169. MR 44 \#5426.

6.- Borel measurable mappings for nonseparable metric spaces, Dissertation, University of Rochester, Rochester, N. Y., 1969.

7. F. Hausdorff, Mengenlehre, 3rd ed., de Gruyter, Berlin, 1937; English transl., Chelsea, New York, 1957. MR 19, 111.

8. S. T. Hu, Theory of retracts, Wayne State Univ. Press, Detroit, Mich., 1965. MR 31 \#6202.

9. K. Kuratowski, Topologie. Vol. 1, 4th ed., PWN, Warsaw, 1958; English transl., Academic Press, New York; PWN, Warsaw, 1966. MR 19, 873; 36 \#840.

10._, Topologie. Vol. 2, 3rd ed., Monografie Mat., Tom 21, PWN, Warsaw, 1961; English transl., Academic Press, New York; PWN, Warsaw, 1968. MR 24 \# A2958; 41 \#4467.

11._, Quelques problèmes concernant les espaces métriques non-séparables, Fund. Math. 25 (1935), 545.

12.—, Sur le prolongement de l'homéomorphie, Comptes Rendus Hebdomadaires des Séances de l'Académie des Sciences, 197 (1933), 1090.

13. A. H. Stone, Non-separable Borel sets, Rozprawy Mat. 28 (1962), 41 pp. MR 27 \#2435.

14._, Borel and analytic metric spaces, Proc. Washington State Univ. Conf. on General Topology (Pullman, Wash., 1970), Pi Mu Epsilon, Dept. of Math., Washington State Univ., Pullman, Wash., 1970, pp. 20-33. MR 42 \#3745.

Department of Mathematics, University of Connecticut, Storrs, Connecticut 06268 